\title{
MODELING GROWTH IN ARM CIRCUMFERENCE OF INFANTS IN JIMMA TOWN, SOUTH-WEST ETHIOPIA
}

\author{
Sileshi Fanta \\ Department of Statistics, Faculty of Science, Addis Ababa University, PO Box 1176 \\ Addis Ababa, Ethiopia, E-mail: fantasil@yahoo.com
}

\begin{abstract}
The paper models the growth in arm circumference of 450 infants during their first year of life. The model is based on longitudinal data obtained from Jimma, a town in southeast Ethiopia. A linear mixed model was employed to see how arm circumference of these infants change over their first year of life. A polynomial model with third degree term is found to be significant. The Loess smoothing technique is applied to check whether our model fit the data set well. The fitted arm circumference is very close to the smoothed curves. Three dropout models are fitted and compared using the likelihood ratio statistic. The results favour the Missing at Random (MAR) process. This makes the likelihood-based analysis justifiable. Our final model is dominated by the linear term early in life, with contribution of the quadratic and cubic terms as the child ages a few months. This indicates that arm circumference growth stops for several months, and possibly there are losses in arm circumferences. This phenomenon can be attributed to malnutrition.
\end{abstract}

Key words/phrases: Loess, MAR, MCAR, MNAR

\section{INTRODUCTION}

A longitudinal community based study which focused on infant survival was done in the Jimma, Illubabor and Keffecho Administrative zones in southwest Ethiopia in 1992-1994. With the aim of identifying all live births, all traditional birth attendants in the above areas were involved in the fieldwork. Traditional birth attendants are women residents in the area who serve and assist women during pregnancy and delivery. They have easy access to women in the fertile age group, and were able to assess the status of pregnancy. Each traditional birth attendant was given responsibility to attend about 300 households, and went house to house regularly to locate pregnant women who were in their second trimester. The traditional birth attendants reported daily in person to the enumerator responsible for her Kebele (a subtown). For each one of the three Kebeles one enumerator was assigned. The enumerator monitored the expectant mother so as to reach her on time soon after delivery. For each of three or more enumerators one supervisor (mainly nurses) was assigned who checked and supported data collection (Lesaffre et al., 1999).

In each Kebele all one-year live-birth cohorts were recruited for the study and followed for one year from 1992 to 1994. Soon after birth data on infant anthropometrics and mothers' socioeconomic variables were collected. Then regular follow-up about the infants was made bimonthly until their first birthday or to an earlier death. Seven visits were made during the data collection process. The data considered in this study refer only to 450 randomly selected infants.

The growth in arm circumference is our subject of interest in this study because it can be used to measure the status of a child's nutrition. Arm circumference could help us to know the thickness of the arms of a healthy child through which the nutrition status can be approximated. Measurement of arm circumference can easily be done by putting a tape measure around a child's upper arm. A healthy child will have thick arms and a large arm circumference. A thin malnourished child will have thin arms and a small arm circumference. Healthy arm circumference for age line starts at $10.5 \mathrm{~cm}$, which is the arm circumference of a healthy child at birth. The age line rises steeply from birth on and by the time a child is one year old, the arm circumference is nearly $16 \mathrm{~cm}$ (King et al., 1973). In other words, a child's arm gets much thicker during the first year of life. During the next four years of a child's life that is from the time the child is one up to five years of age, the graph of the age line is nearly flat. A child's arm circumference, therefore, only grows by a little over one centimetre from the time it is one until the time it is five.

As mentioned in King et al. (1973), a child is considered as malnourished if its arm circumference is below $14 \mathrm{~cm}$ at birth. This marks the difference between healthy and malnourished 
children. From the above ideas one can conclude that arm circumference is one important measure in monitoring normal child growth. The objective of this paper is then to model the growth in arm circumference of infants in Jimma town.

\section{MATERIALS AND METHODS}

\section{Data sources, description and models}

The data are obtained from Jimma Infant Survival Project done from 1992-1994. The arm circumference of infants as well as other anthropometrics measurements were measured for all infants at approximately $0,2,4,6,8,10$ and 12 months of age; zero stands for time of birth. The minimum and maximum number of visits per infant is 1 and 7 , respectively. The data considered in this paper are based on 450 randomly selected subjects, which comprise 2794 observations. The response variable used is arm circumference. The covariates considered in the paper are age of mother at first visit, size of the household, marital status of mothers, educational level of mothers, number of antenatal visits, month of birth and sex of child.

Our data consisted of repeated measurements of the same subjects over time. It is then natural to find a methodology that will apply for the type of data at hand. The modelling methods that work for such data are mixed-effects models.

Mixed effects models provide a powerful and flexible tool for analyzing grouped data, that is, data that can be classified according to one or more grouping variables. Such models typically describe relationships between a response variable and some covariates in data grouped according to one or more classification factors. One of the areas of application for mixed models is in longitudinal data, and as our data are longitudinal type, these methods fulfil our need.

Since change in arm circumference, which is a continuous response variable, is the main object of the study, it is of interest first to study mean effect of time alone on the arm circumference. To achieve our objective we used a linear mixed model.

In a linear mixed model, the repeated measurements are modelled using a linear regression model, with parameters that are allowed to vary over individuals, and which are therefore called random effects or subject-specific regression coefficients. Their mean reflects the average evolution in the population, and therefore they are called the vector of fixed effects (Verbeke and Molenberghs, 1997).

\section{Selection of a preliminary mean structure}

The plot of individual growth curve in Figure 1 and the Loess smoothed curve in Figure 2 suggest that a polynomial of degree two seems adequate to explain the arm circumference as a function of age. These preliminary informal analyses also indicate that the intercept and slope are different for different infants. Therefore, having a different slope for each infant leads to subject specific regression coefficients, which represent the random effect in our linear mixed model. Our provisional model for arm circumference with time (age in months) as explanatory variable is:

$$
\begin{aligned}
Y_{i j}= & \beta_{0 i}+\beta_{1 i} t_{i j}+\beta_{2 i} t_{i j}^{2}+\varepsilon_{i j} \\
& i=1, \ldots, 450 ; \quad j=1, \ldots, \mathrm{n}_{\mathrm{i}}
\end{aligned}
$$

where

$t_{i j}$ is age of child $i$ at the $j$ th measurement

$Y_{i j}$ is arm circumference of child $i$ measured at age $t_{i j}$

$\beta_{0 i}$ represents the mean arm circumference of child $i$ at birth

$\beta_{1 i}$ is the linear time effect on child $i$

$\beta_{2 i}$ is the quadratic time effect on child $i$

$\varepsilon_{i j}$ is the mean zero deviation representing the within-infant variability

$n_{i}$ is the number of measurements on child $i$

We assume that $\beta_{0}, \beta_{1}$, and $\beta_{2}$ are the average intercept, linear and quadratic time effect of the population. After correcting for the effect of individual characteristics the individual coefficients can then be expressed as:

$$
\beta_{0 \mathrm{i}}=\beta_{0}+\mathrm{b}_{0 \mathrm{i}}, \beta_{1 \mathrm{i}}=\beta_{1}+\mathrm{b}_{1 \mathrm{i},}, \beta_{2}=\beta_{2}+\mathrm{b}_{2 \mathrm{i}}
$$

The error terms $b_{0 i}, b_{1 i}$ and $b_{2 i}$ represent the random effect on child $i$ corresponding to the intercept, the linear and quadratic time effects, respectively. The parameters $\beta_{0}, \beta_{1}$ and $\beta_{2}$ are assumed to be the same for all infants. Therefore, model (1) can be rewritten as follows:

$Y_{i j}=\left(\beta_{0}+b_{0 i}\right)+\left(\beta_{1}+b_{1 i}\right) t_{i j}+\left(\beta_{2}+b_{2 i}\right) t_{i j}^{2}+\varepsilon_{i j}$

or equivalently as

$$
Y_{i j}=\beta_{0}+\beta_{1} t_{i j}+\beta_{2} t_{i j}^{2}+b_{0 i}+b_{1 i} t_{i j}+b_{2 i} t_{i j}^{2}+\varepsilon_{i j}
$$

The matrix form of (2) is 


$$
Y_{i}=Z_{i} \beta+Z_{i} \mathbf{b}_{i}+\varepsilon_{i}
$$

where

$$
\begin{aligned}
& Y_{i}=\left[\begin{array}{c}
Y_{i 1} \\
Y_{i 2} \\
\cdot \\
\cdot \\
\cdot \\
Y_{i n_{i}}
\end{array}\right] \quad Z_{i}=\left[\begin{array}{ccc}
1 & t_{i 1} & t_{i 1}^{2} \\
1 & t_{i 2} & t_{i 2}^{2} \\
\cdot & \cdot & \cdot \\
\cdot & \cdot & \cdot \\
\cdot & \cdot & \cdot \\
1 & t_{i n_{i}} & t_{i n_{i}}^{2}
\end{array}\right] \varepsilon_{i}=\left[\begin{array}{c}
\varepsilon_{i 1} \\
\varepsilon_{i 2} \\
\cdot \\
\cdot \\
\cdot \\
\varepsilon_{i n_{i}}
\end{array}\right] \\
& \beta=\left(\beta_{0}, \beta_{1}, \beta_{2}\right)^{\prime} \quad \mathrm{b}_{\mathrm{i}}=\left(\mathrm{b}_{0 \mathrm{i}}, \mathrm{b}_{1 \mathrm{i}}, \mathrm{b}_{2 \mathrm{i}}\right)^{\prime}
\end{aligned}
$$

Model (3) is a linear mixed model with fixed effects $\beta$ and random effect $b_{i}$.

To make inferences we need to make assumptions about the probability distribution of these random components. The assumptions are:

1. The vector of random effects $b_{i}$ is normally distributed with mean vector 0 and a $3 \times 3$ covariance matrix $\mathbf{D}$. That is, $b_{i} \sim N(0, D)$,

where $\mathbf{0}=(0,0,0)^{\prime}$ and $\mathbf{D}=\left[\begin{array}{lll}d_{11} & d_{12} & d_{13} \\ d_{21} & d_{22} & d_{23} \\ d_{31} & d_{32} & d_{33}\end{array}\right]$.

2. The vector of residual component $\varepsilon_{i}$ is normally distributed with mean vector $\mathbf{0}$ and $\left(\mathrm{n}_{\mathrm{i}} \times \mathrm{n}_{\mathrm{i}}\right)$ covariance matrix $\Sigma_{\mathrm{i}}$.

3. Between-infant measurements are assumed uncorrelated. That means $b_{1} b_{2} \ldots b_{450} \varepsilon_{1} \varepsilon_{2} \quad \varepsilon_{450}$ are assumed to be independent.

Therefore, the marginal distribution of the vector of responses $Y_{i}$ is normally distributed with mean vector $\mathrm{Z}_{\mathrm{i}} \beta$ and covariance matrix $V_{i}=Z_{i} D Z_{i}^{\prime}+\Sigma_{i}$. That is $\mathrm{Y}_{\mathrm{i}} \sim \mathrm{N}\left(Z_{i} \beta, Z_{i} D Z_{i}^{\prime}+\Sigma\right)$

\section{RESULTS AND DISCUSSION}

\section{Exploratory data analysis}

Our data set consists of a number of observations on many subjects with some explanatory variables and missing values. Primarily we tried to get the overall features of the data using exploratory data analysis. The exploratory phases are useful to select an appropriate statistical model.

\section{Exploring the mean structure}

To visualize the pattern of the data, plots of arm circumference against time were constructed. In Figure 1 observations for 30 randomly selected subjects were joined to investigate how arm circumference changes over time. It is noticeable from the plots that arm circumference is not constant as time evolves. Furthermore evidence of variability between and within individuals is observed. Infants also do not maintain their relative size of arm circumference over time. Incomplete profiles correspond to either dropouts or death.

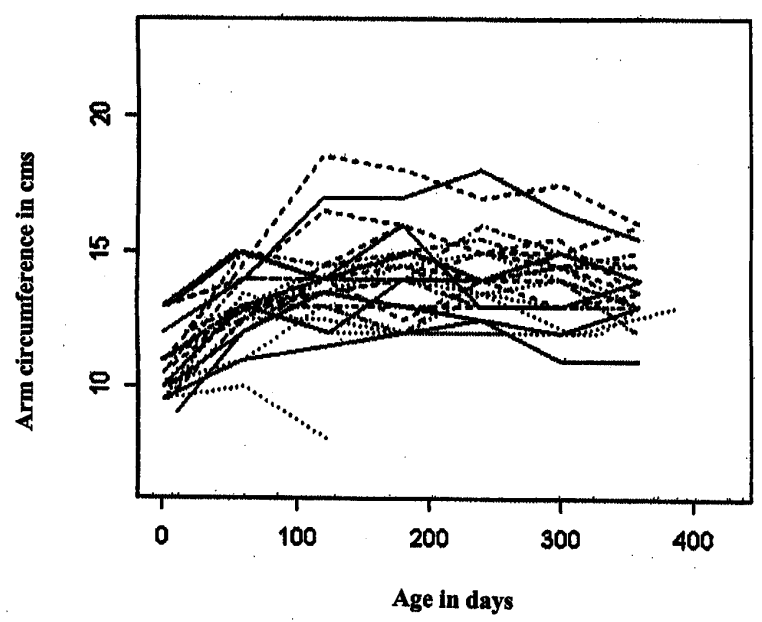

Fig. 1. Profile plot of arm circumference against time for 30 randomly selected infants.

By looking at the profile plot (Figure 1), it is difficult to guess the most appropriate functional form of dependence of the mean arm circumference on time. We use the scatter-plotsmoother to show the functional dependence without imposing parametric assumptions about the dependence. This non-parametric curve is displayed in Figure 2. The curve is obtained using the Loess method in S-PLUS software.

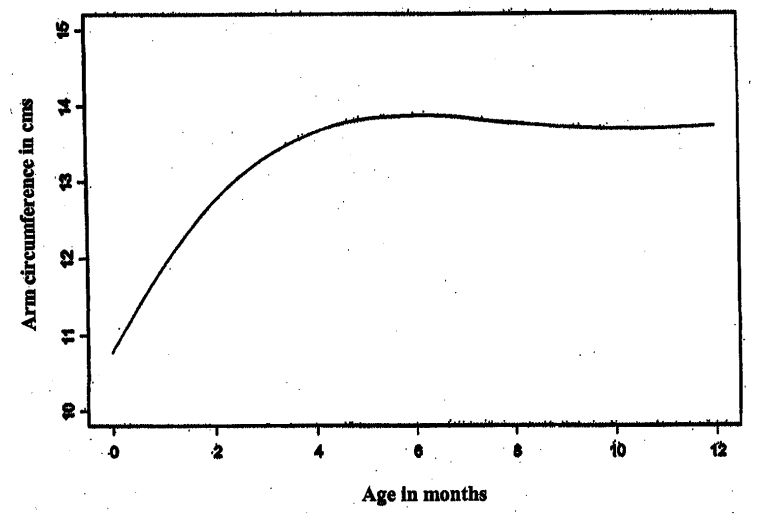

Fig. 2. Loess smooth curve of arm circumference against time. 
Figure 2 shows a sharp increase of the estimated mean response profile of arm circumference from the beginning up to the age of about four months, and thereafter the increase slows down. This curve suggests that the true statistical relationship between arm circumference and age is curvilinear. The pattern suggests that it might be reasonable to approximate the relationship by a quadratic function of time.

\section{Exploring the covariance structure}

The inferential focus of this study is on the mean response of arm circumference. The covariance structure is of secondary interest, but must be accommodated in the analysis of the data to ensure valid inferences about the structure of mean arm circumference.

In this type of longitudinal study there are at least three possible components of variability, namely random effects, serial correlation and measurement error (Diggle et al., 1994). Random effects are effects that arise from the characteristic of individual infants. Therefore, these effects explain the stochastic variation between infants. On the other hand, measurements of arm circumference on successive occasions of the same infant are most likely to be serially dependent. Hence, we cannot extract as much information from these dependent observations as we could extract from the same number of independent measurements. That is, serial correlations mask part of the within-infant variation in the data. Finally, because arm circumferences are determined using a measuring tape during data collection, it is natural to expect the existence of measurement error. Therefore, in our further analysis we will look for these three sources of variability.

Since in covariance structure models all the variability in the data cannot be explained by explanatory variables (risk factors), we start to explore the covariance structure by first removing all systematic trends (Verbeke and Molenberghs, 1997). Hence, residuals are obtained after regressing arm circumference on potential covariates and their interaction with time and time squared (PROC GLM procedure in SAS).

Figure 3 displays the estimated average evolution of the variance of the data at each time point. From the plot we can see that the variance is not constant. It shows an increasing tendency up to about the age of six months, and a slow decrease after the age of six months.

To gain insight into the nature of the association among repeated measurements of arm circumfer- ence within subjects, we considered the scatter plot matrix of the residuals as shown in Figure 4. The scatter plot is made by discretizing time points by visit number (every other month). The upper panel of this Figure gives the correlation matrix of residuals for visits 1 to 7 . In general, it shows that there is a decreasing tendency of correlation as the observations are separated further apart in time. On the other hand the scatter plots have a circular shape indicating that serial correlation is not very strong.

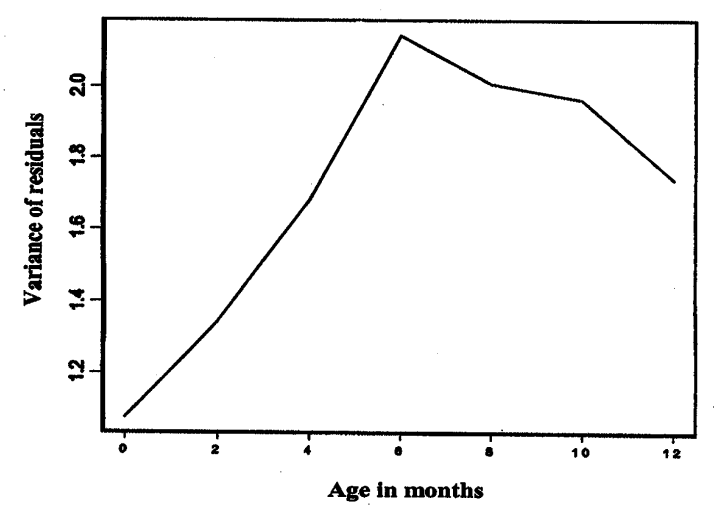

Fig. 3. Plot of the variance of residuals against time

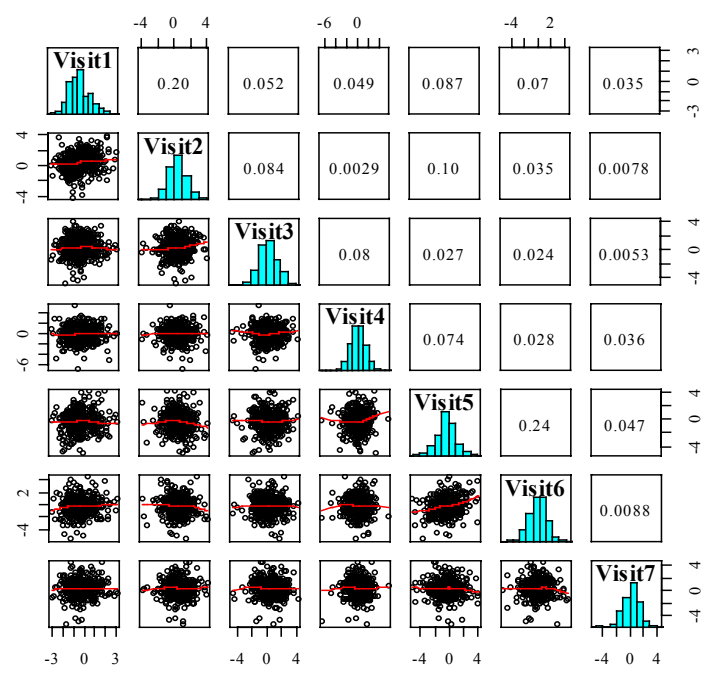

Fig. 4. Scatter plot and correlation matrices of residuals by visit number.

\section{Parameter estimates and adequacy of the model}

\section{Parameter estimates}

This section deals with selection of random effect, selection of covariance structure and estimation and inference about the dependence of arm circumference over time. The confirmatory statistical hypothesis test procedures are used. 


\section{Selection of the random effect}

Our objective in this section is to decide whether or not to include the three random effects (random intercept, linear random slope, quadratic random slope) in the model. For the present we use the most elaborated model we are prepared to contemplate for the mean arm circumference. At the beginning we fitted a linear mixed model assuming the diagonal elements in the covariance matrix $\Sigma_{\mathrm{i}}$ are all equal and the off-diagonal elements are zero. Therefore, the variance of the response vector $Y_{i}$ depends on time, only through the component $Z_{i} D Z_{i}^{\prime}$.

We followed a hierarchical test procedure to see if the quadratic random time effect is significant. If significance is confirmed then linear random time effect and random intercept will also be included. Hence our tests begin with the inquiry whether or not the quadratic time effects differ between infants. The formulation of the test of hypothesis at a specified $\alpha$-level of significance is:

$H_{0}: \mathrm{d}_{13}=\mathrm{d}_{23}=\mathrm{d}_{33}=0$ against the alternative $H_{\mathrm{a}}$ : at least one of the $\mathrm{d}_{\mathrm{i} 3}$ is different from $0, \mathrm{i}=1,2,3$.

In the above $d_{13}, d_{23}, d_{33}$ are: the covariance of the random intercept and quadratic random slope, the covariance of the linear random slope and quadratic random slope, and the variance of the quadratic random slope, respectively.

The likelihood ratio test cannot be used for testing this null hypothesis because the likelihood ratio test suffers from a boundary problem under the null hypothesis $d_{33}=0$. Ignoring the boundary problems may result in too parsimonious covariance structures. This correction due to the boundary problem reduces the $\mathrm{p}$-values in order to protect against the use of oversimplified covariance structures (Verbeke and Molenberghs, 2000). To avoid this boundary value problem we applied the asymptotic mixture of chi-squared distributions for the likelihood ratio test statistic. This statistic is the difference of minus twice the logarithm of the likelihoods under the null and the alternative hypothesis. A large value of this difference rejects the null hypothesis in favour of the alternative hypothesis that there is a significant improvement in the fit when the extra random effect parameters are included. The following four random effect models were considered for testing.

Model 1: Intercept, linear in time, time squared;

Model 2: Intercept, linear in time;

Model 3: Intercept only;

Model 4: Without any random effect.
The likelihood ratio test statistics based on maximum likelihood (ML) and restricted maximum likelihood (REML), together with the corresponding $\mathrm{p}$-values are displayed in Table 1 . From the results we can see that all observed values of the test statistics are very large, yielding $p$-values less than 0.0001 . We conclude that the covariance structure should not be simplified by deleting the quadratic random effects from the model.

Although this analysis is performed by including all potential covariates and their interaction with time and time squared in the mean structure of the model, the same result was obtained by using only time as covariate. In both cases, the covariance structure cannot be simplified any further by deleting the quadratic random effect from the model.

Table 1. Likelihood ratio test for random effects using maximum as well as restricted maximum likelihood estimation. (All potential risk factors were included in the model).

\begin{tabular}{|c|c|c|c|c|c|c|c|}
\hline \multirow[t]{2}{*}{$\begin{array}{l}\text { Random } \\
\text { Effects }\end{array}$} & \multicolumn{2}{|c|}{-2 loglikelihood } & \multicolumn{2}{|c|}{$\begin{array}{c}\text { LR-test } \\
\text { Statistics }\end{array}$} & \multirow{2}{*}{$\begin{array}{c}\text { Chi- } \\
\text { Square } \\
\chi_{k 1: k 2}\end{array}$} & \multicolumn{2}{|c|}{ P-value } \\
\hline & ML & REML & ML & REML & & ML & REML \\
\hline & 8133.9 & 8332.2 & & & - & & \\
\hline & 8257.0 & 8456.3 & 123.1 & 124.1 & $\chi_{2: 3}$ & & 0.000 \\
\hline Mode & 8500.4 & 8704.0 & 243.4 & 247.7 & $\chi_{1: 2}$ & & $<0.0001$ \\
\hline Model 4 & 9549.7 & 9753.7 & 1049.3 & 1049.7 & $\chi_{0: 1}$ & & $<0.0001$ \\
\hline
\end{tabular}

The P-value is calculated by giving equal weights for mixture of two chi-squared distribution with $\mathrm{k} 1$ and $\mathrm{k} 2$ degrees of freedom

. That is $\mathrm{P}$-value $=\mathrm{P}\left(\chi_{\mathrm{k} 1 \mathrm{k} 2} \geq\right.$ LRT Statistics $)=0.5 \mathrm{P}\left(\chi_{\mathrm{k} 1} \geq \mathrm{LRT}\right.$ Statistics $)+0.5 \mathrm{P}\left(\chi_{\mathrm{k} 2} \geq\right.$ LRT Statistics $)$.

\section{Selection of the covariance structure}

In the previous section we selected the random effects based on the assumption that random effects account for most of the variation in the data and the remaining error components $\varepsilon_{i}$ have a simple covariance structure. In other words the covariance matrix $\Sigma_{\mathrm{i}}=\sigma^{2} I_{n i}$ where $I_{n i}$ is the identity matrix of dimension $n_{\mathrm{i}}$. This implies that the within-infant measurements of arm circumference are independent and have constant variance $\sigma^{2}$. In what follows we extend this assumption and search for a different type of covariance structure. We investigate in a more confirmatory way for the general linear mixed model (3). The covariance matrix in (3) was expressed by $V_{i}=Z_{i} D Z_{i}^{\prime}+\Sigma_{\mathrm{i}}$ where $\mathbf{D}$ is the covariance matrix of random effects $b_{i}$ and $\Sigma_{i}$ is the covariance matrix of $\varepsilon_{i}$. The error term encompasses both measurement error and serial correlation. Here by keeping the covariance matrix D unstructured, we will find the best structure for $\Sigma_{\mathrm{i}}$.

The likelihood ratio test cannot be used to differentiate between models with different covariance structures, if these models are not 
nested to each other. On the other hand SAS procedure PROC MIXED allows to fit mixed models of different types of covariance structure but it only provides information criteria to select between such models; here we use Akaike's Information Criteria (AIC). The AIC is based on decision theory and penalizes the log-likelihood by subtracting the number of variance components in the model. The model, which has the smallest AIC in absolute value, will be considered the 'best' model. It should be strongly emphasized that information criteria only provide rules of thumb to discriminate between several statistical models. They should never be used or interpreted as formal statistical tests of significance (Verbeke and Molenberghs, 2000). The approach used for selection of the covariance structure is to fit several structures and compare them using AIC. The values of the AIC for some of the covariance structures considered are displayed in Table 2. From the table the AIC are in favour of a heterogeneous first order autoregressive (ARH (1)) covariance structure. However, all of these models, except the one with simple covariance structure, suffered from not having a positive definite covariance matrix. The estimates for elements of matrix $\mathbf{D}$ are displayed in Table 3.

Table 2. The Akaike's Information Criteria (AIC) obtained by fitting models with different covariance structures.

\begin{tabular}{ll}
\hline Covariance Structure & AIC \\
\hline Simple & -4165.9 \\
First order autoregressive & -4009.2 \\
Power & -4009.2 \\
Exponential & -4009.2 \\
Gaussian & -4121.4 \\
ARH (1) & -4001.9 \\
\hline
\end{tabular}

From the table we can see that the estimate for $d_{11}$ for three of the models is zero. The estimates for $d_{33}$ are also very close to zero. SAS procedure PROC MIXED also reported that the estimated $\mathbf{D}$ matrix is not positive definite for all of covariance structures considered with the exception of the simple covariance structure. This could undermine the hierarchical model interpretation of our data.

Even though the simple covariance structure is inferior in terms of AIC, it is the only structure with positive definite covariance matrix. In addition to that, there is no strong evidence of serial correlation (even before consideration of random effects) from our exploratory analysis (Fig. 4). Therefore, keeping the covariance matrix D unstructured we use the simple covariance structure for the remaining error component.

While selecting the random effects, the simple covariance structure was used and, therefore we are forced to select the same. Hence there is no need to check for random effect again, and as a result the random quadratic time effect will be used. However, we need to test for the mean structure of the model we have adopted. The next step is whether we can build a polynomial model of degree 3 or more or whether we can simplify the model by using a linear rather than a quadratic time effect. By fitting models with and without cubic effect, we get a value for the likelihood ratio statistic, which is equal to 432.2 with one degree of freedom. This corresponds to a p-value of less than 0.0001. The F-test (Table 4) also yields similar results. We therefore, include the cubic time effect in our model.

A polynomial regression model with fourth degree term was also tested; but its effect is found to be insignificant (results not shown). The tests for random effects also retain the quadratic random effect. The restricted maximum likelihood estimates (REML) and standard errors for the fixed effects well as the variance components of the fitted model are presented in Table 5. Therefore, on the basis of the above considerations, the fitted model can be stated as:

$$
\hat{Y}_{i j}=10.79+1.27 t_{i j}-0.17 t_{i j}^{2}+0.007 t_{i j}^{3}
$$

where $t$ is measured in months.

From this we can see that arm circumference is dominated by the linear term early in life, with contribution of the quadratic and cubic terms as the child ages a few months. This follows the smooth estimated population growth curve (Fig. 2) indicating that arm circumference stops growing for several months. Possibly there could be losses in circumferences. This observation is an indication that the cause for the losses could be due to malnutrition. This incidence started to take place after the age of about 4 months - a time when infants need to be fed additional solid food.

\section{Adequacy of model}

The suitability of the statistical model for the data should be examined before inferences based on the model are made. What we have done in our case is take up this issue after performing inferences. We did this just for the sake of convenience in reporting. In this section we only try to check the assumptions made about the dropouts and make some informal checks for the model goodness-of-fit. 
Table 3. The Covariance parameter estimates $\left(d_{i j}=\right.$ elements of matrix $\left.D\right)$ for the different covariance structures.

\begin{tabular}{lrrrrrr}
\hline Covariance Structure & \multicolumn{1}{c}{$d_{11}$} & \multicolumn{1}{c}{$d_{21}$} & $d_{22}$ & \multicolumn{1}{c}{$d_{31}$} & \multicolumn{1}{c}{$d_{32}$} & $d_{33}$ \\
\hline Simple & 0.52770 & -0.00994 & 0.07926 & -0.00135 & -0.00483 & 0.000334 \\
First order autoregressive & 0 & 0.06707 & 0.02689 & -0.00401 & -0.00100 & $2.027 \mathrm{E}-6$ \\
Power & $2.34 \mathrm{E}-18$ & 0.06707 & 0.02689 & -0.00401 & -0.00100 & $2.029 \mathrm{E}-6$ \\
Exponential & 0 & 0.06706 & 0.02689 & -0.00401 & -0.00401 & $2.019 \mathrm{E}-6$ \\
Gaussian & $1.65 \mathrm{E}-17$ & 0.07263 & 0.02646 & -0.00445 & -0.00100 & $5.61 \mathrm{E}-21$ \\
ARH (1) & 0 & 0.10970 & 0.02335 & -0.00749 & -0.00081 & 0 \\
\hline
\end{tabular}

Table 4. Tests for fixed effects.

\begin{tabular}{lllll}
\hline Effect & $\begin{array}{l}\text { Numerator degree of } \\
\text { Freedom }\end{array}$ & $\begin{array}{l}\text { Denominator degree of } \\
\text { freedom }\end{array}$ & F-test Statistics & P-value \\
\hline Linear time effect & 1 & 436 & 1339.13 & $<0.0001$ \\
Quadratic time effect & 1 & 414 & 680.91 & $<0.0001$ \\
Cubic time effect & 1 & 1491 & 400.73 & $<0.0001$ \\
\hline
\end{tabular}

Table 5. REML estimates for the parameters of the fitted mode.

\begin{tabular}{|c|c|c|c|}
\hline Effect & Parameter & Estimates & Standard Error(Sandwich) \\
\hline Intercept & $\beta_{0}$ & 10.7892 & 0.05024 \\
\hline Linear Time Effect & $\beta_{1}$ & 1.2654 & 0.03452 \\
\hline Quadratic Time Effect & $\beta_{2}$ & -0.1659 & 0.00635 \\
\hline Cubic Time effect & $\beta_{3}$ & 0.0067 & 0.00033 \\
\hline \multicolumn{4}{|l|}{ Covariance of $b_{i}$} \\
\hline Variance $\left(\mathrm{b}_{0 \mathrm{i}}\right)$ & $d_{11}$ & 0.6724 & 0.0717 \\
\hline Variance $\left(b_{1 i}\right)$ & $\mathrm{d}_{22}$ & 0.1090 & 0.0121 \\
\hline Variance $\left(\mathrm{b}_{2 \mathrm{i}}\right)$ & $d_{33}$ & 0.0005 & 0.0001 \\
\hline Covariance of $\left(\mathrm{b}_{0 \mathrm{i}}, \mathrm{b}_{1 \mathrm{i}}\right)$ & $\mathrm{d}_{12}=\mathrm{d}_{21}$ & -0.0410 & 0.0223 \\
\hline Covariance of $\left(\mathrm{b}_{0 \mathrm{i}}, \mathrm{b}_{2 \mathrm{i}}\right)$ & $\mathrm{d}_{13}=\mathrm{d}_{31}$ & 0.0007 & 0.0016 \\
\hline Covariance of $\left(b_{1 i}, b_{2 i}\right)$ & $\mathrm{d}_{23}=\mathrm{d}_{32}$ & -0.0069 & 0.0009 \\
\hline \multicolumn{4}{|l|}{ Covariance of $\varepsilon_{i}$} \\
\hline Variance of $\varepsilon_{\mathrm{i}}$ & $\sigma^{2}$ & 0.4824 & 0.0175 \\
\hline
\end{tabular}

\section{Missing data issues}

Initially, it was planned to measure arm circumference in seven visits (every other month starting from birth). However, we did not succeed to have the same number of measurements for all infants. Some data are simply missing or some infants have measurements for fewer than seven occasions.

Here our focus of attention is on the dropouts, where a child is observed until a certain point in time, thereafter no measurements are taken. Therefore, we will investigate the dropout process and check whether it affects our earlier analyses concerning the mean arm circumference profiles, performed by PROC MIXED procedure in SAS.

A dropout process is said to be missing completely at random (MCAR) if the dropout is independent of both unobserved and observed data, and missing at random (MAR) if, conditional on the observed data, the dropout is independent of unobserved measurements. If the dropout process depends on unobserved measurements, the process is termed as missing not at random (MNAR). If a dropout process is random, then valid analysis can be done through a likelihood based analysis that ignores the dropout mechanism, provided the parameters describing the measurement process are functionally independent of the parameters describing the dropout process, the so called parameter distinctness condition. That is, when the parameters describing the measurement process and missing process are distinct, within the likelihood framework ignorability is equivalent to having both MAR and MACR (Verbeke and Molenberghs, 2000). In this case a valid analysis can be performed using SAS procedure PROC MIXED, provided the order of measurements is correctly specified.

We will now explore the missing data process by assuming that the dropout probability at occasion $j$ depends on both the current outcome $Y_{i j}$ and the previous one $Y_{\mathrm{ij}-1}$. This leads to the following model 


$$
\ln \left(\frac{P\left(R_{i j}=0 \mid y_{i}\right)}{1-P\left(R_{i j}=0 \mid y_{i}\right)}\right)=\psi_{0}+\psi_{1} y_{i j}+\psi_{2} y_{i j-1}
$$

with $\quad R_{i j}=\left\{\begin{array}{l}1 \text { if } Y_{i j} \text { is } \begin{array}{r}\text { observed } \\ 0\end{array}\end{array}\right.$

Observe that $P\left(R_{i j}=0 \mid y_{i}\right)$ is the conditional probability of a subject $i$ dropping out at time $j$ depending on the current outcome $Y_{i j}$ and previous outcome $Y_{\mathrm{ij}-1}$.

The whole sample of 450 subjects considered in this study is used to estimate the parameters. The assumption considered here is that the relationship among the measurements from a subject are the same whether or not some of these measurements are unobserved due to non-response. It is this assumption that allows us to infer something about the informativeness of the non-response process (Verbeke and Molenberghs, 1997). If the value of $\psi_{1}$ is non-zero, then the dropout process depends on the unobserved measurements and it is a MNAR model. If $\psi_{1}$ is zero and $\psi_{2}$ is different from zero, then it is a MAR model that depends on previous measurements. We assume the dropout process to be MCAR when both $\psi_{1}$ and $\psi_{2}$ are zero.

The results of these three models, obtained from OSWALD Software in S-PLUS, are summarized in Table 6. The time effect with a polynomial of degree three is incorporated in the mean structure of the linear mixed model.

Table 6. Parameter estimates and likelihood analysis of the dropout process.

\begin{tabular}{lrrr}
\hline Parameter & Dropout Modeled & \\
& MCAR & MAR & MNAR \\
\hline Intercept & 10.860 & 10.786 & 10.789 \\
Linear time effect & 1.065 & 1.284 & 1.258 \\
Quadratic time effect & -0.121 & -0.169 & -0.164 \\
Cubic time effect & 0.004 & 0.007 & 0.007 \\
Dropout Parameters & & & \\
$\psi_{0}$ & -3.235 & 0.903 & -0.227 \\
$\psi_{1}$ & 0 & 0 & -0.181 \\
$\psi_{2}$ & 0 & -0.329 & -0.049 \\
-2 loglikelihood & 23050.96 & 22926.44 & 22923.82 \\
\hline
\end{tabular}

The likelihood ratio test statistic used to compare MAR with MCAR is 124.52 with one degree of freedom and the corresponding $\mathrm{p}$-value is less than 0.0001. This rejects the MCAR assumption, the dependence of the probability of the dropouts on the previous observed measurements. The comparison between MAR and MNAR yields a likelihood ratio statistic of 2.61 with one degree of freedom. This corresponds to a p-value of 0.106, favouring the assumption of MAR. Based on the above suggestions, performing a likelihood ignorable analysis in SAS procedure PROC MIXED is justifiable.
We can, therefore, analyze all available information without the need neither to delete nor to impute measurements or the entire subjects.

\section{Model checking}

Some graphical techniques were applied to informally check whether our models fit the data set well. Our models are the models for the mean and covariance structure of the data. Therefore, it is necessary to compare the fitted and observed mean response profiles of arm circumference as well as the model based and sample variance functions.

Loess smoothing technique is applied to summarize the trend of average arm circumference as a function of time. This technique estimates the underlying regression function without any restrictive parametric form. In addition to its use in assisting to choose the parametric models, it can also be used as diagnostic tool by comparing the parametric and non-parametric fits.

The superimposed fitted average profile on the smoothed Loess curves (two different bandwidths) are indicated in Figs 5a and 5b. Figure 5a compares Loess fit (smoothing parameter $=0.45$ ) with our fitted average arm circumference. Figure $5 \mathrm{~b}$ compares Loess fit (smoothing parameter $=0.8$ ) with the fitted mean arm circumference. From these plots we can see that the fitted average profile is very close to the smoothed curves. However, the discrepancy gets larger after the age of 8 months. This could be either due to relatively small number of measurements considered after the age of 8 months or it could be due to the covariance structure considered.

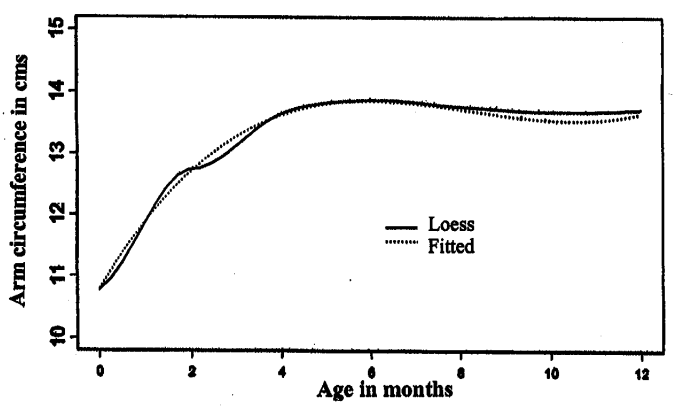

Fig. 5a. The fitted average profiles of arm circumference (small bandwidth).

The other thing to do was to check the appropriateness of the selected covariance structure informally. Therefore, the model-based fitted variances are compared with the sample variances, which are based on the observed data. The model-based variance at time $t$ is given by: 


$$
\operatorname{Var}\left(\hat{Y}_{i}(t)\right)=\left(1, t, t^{2}\right) \hat{D}\left(1, t, t^{2}\right)^{\prime}+\hat{\sigma}^{2}
$$

Substituting the estimated values of the covariance matrix of the random effects $\mathrm{D}$ and the residual variance $\sigma^{2}$ reported in Table 4 , we have the following fitted variance function:

$$
\operatorname{Var}\left(\hat{Y}_{i}(t)\right)=1.1548-0.082 t+0.1104 t^{2}-0.0138 t^{3}+0.0005 t^{4}
$$

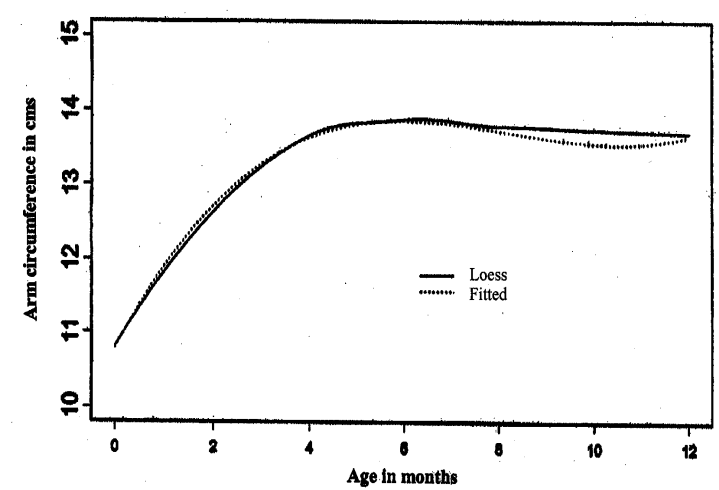

Fig. $5 b$. The fitted average profiles of arm circumference (large bandwidth).

The plot of this function together with the sample variance of residuals is displayed in Figure 6. Both variance functions show similar trends up to the age of 6 months. Beyond 6 months there is deviation between these variance functions. This could be due to the fact that a relatively small amount of data was used in the period after 6 months. Nonetheless, the deviation will not create problems on our inference because we employed inferential procedures based on the sandwich estimator for the standard error of the fixed effects. The sandwich estimator yields a consistent estimator of precision even if the covariance model is not correctly specified.

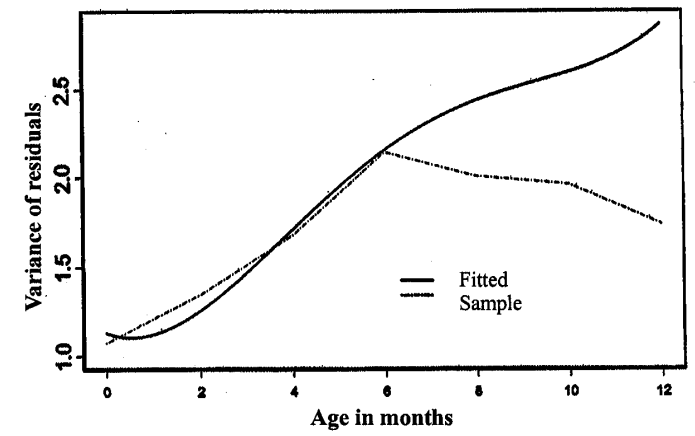

Fig. 6 . The fitted and sample variance function.

\section{INTERPRETATION AND DISCUSSION OF THE RESULTS}

At the beginning some graphical displays were used to have a closer look at the data. Based on these exploratory analyses a preliminary model selection was made. The selected preliminary mean structure was quadratic time effect. Having done that the selection of random effects also resulted in quadratic random effect. While selecting the covariance structure, by keeping the covariance matrix $\mathbf{D}$ of the random term unstructured, a search for the best structure for the covariance of the remaining error component was made. Even though we tried different structures, we ended up in choosing the simple covariance structure. This is due to the assumption that all repeated measures are independent of each other. This means that, the correlation of successive observations is explained only on the unstructured covariance matrix of random effects, and all the variability in the data, which is not taken into account by the random effects, is assumed to be purely measurement error.

Based on the preliminary mean structure a model was fitted and tests for fixed effects were made. A test for polynomial model with third degree term is found to be significant while the fourth degree polynomial was not significant. The test for random effects also resulted in the same quadratic random time effect. Therefore, we changed the model for the mean structure from quadratic time effect to the cubic time effect.

We applied inferential procedures based on the sandwich estimator for the standard error of the fixed effects. The sandwich estimator yielded a consistent estimator of precision, even if the covariance model was not correctly specified. An attempt to informally examine the aptness of our statistical model was made by using the Loess smoothing technique to check whether our model fit the data set well. The fitted arm circumference is very close to the smoothed curves. Three dropout models were fitted and compared with one another using the likelihood ratio statistic. The results favoured Missing at Random (MAR). This suggests that the likelihood-based ignorable analysis is justifiable. Our final model is dominated by the linear term early in life, with contribution of the quadratic and cubic terms as the child ages a few months. 


\section{CONCLUSIONS}

From our modelling process the following conclusions were made. The linear mixed model, which contains only time as explanatory variable, indicates that arm circumference is not constant over the first year of life. The growth of arm circumference increases sharply until the age of about four months and then it slows down thereafter. This incidence is quite completely unusual because normally growth was expected to slow down after the age of one year. This early stop in growth is an indication for malnutrition; of course the growth depends on other covariates considered in the model. The effects of these covariates on our model need further study, and these will be studied in the future.

\section{ACKNOWLEDGEMENTS}

The author would like to thank Dr. Mekonen As efa, Jimma University, Ethiopia for providing the data used in this paper. He is also grateful to Prof. Dr. Geert Molenberghs, Diepenbeek, Belgium for his support and valuable advice.

\section{REFERENCES}

1. Diggle, P.J., Liang, K.Y. and Zeger, S.L. (1994). Analysis of Longitudinal Data. Oxford: Oxford University Press, London.

2. Hastie, T.J. and Tibshirani, R.J. (1990). Generalized Additive Models. Chapman and Hall, New York.

3. King, M.H., King, F.M., Morley D.C., Burgess, H.J. and Burgess, A.P. (1973). Nutrition for Developing Countries. Oxford: Oxford University Press, London.

4. Lesaffre, E., Mekonnen Asefa and Verbeke, G. (1999). Assessing the goodness of fit of the Laired and Ware Model. An example: the Jimma infant survival differential longitudinal study. Statistics in Medicine 18:835-854.

5. Verbeke, G. and Molenberghs, G. (1997). Linear Mixed Models in Practice: A SAS Oriented Approach. Lecture Notes in Statistics 126. New York: SpringerVerlag.

6. Verbeke, G. and Molenberghs, G. (2000). Linear Mixed Models for Longitudinal Data: New York: SpringerVerlag. 